\title{
Developments Towards Correlative Cryo-Light 3D Soft X-Ray Tomography
}

Dunja Skoko, Kenneth Fahy, Paul Sheridan and Tony McEnroe

SiriusXT, Dublin, Dublin, Ireland

SiriusXT is developing a commercial bench-top cryo soft X-ray microscopy system for 3D cryo-soft Xray tomography (cryo-SXT). Our technology facilitates label-free ultrastructural 3D imaging of whole cells up to $10-15 \mu \mathrm{m}$ in diameter, in native state with resolution approaching $30 \mathrm{~nm}$. Image contrast in the 'water window' wavelength range between the K-shell absorption edges of carbon $(284 \mathrm{eV}, \lambda=4.4 \mathrm{~nm})$ and oxygen $(543 \mathrm{eV}, \lambda=2.3 \mathrm{~nm})$ is directly obtained by the higher x-ray absorption by carbon rich structures compared to water.

Great progress has been made over the last two decades in developing cryo-SXT as an imaging technique on synchrotron hosted microscopes [1-4]. Workflows have improved which allow non-synchrotron researchers to access the technique, and significant expertise has been developed in correlating SXT and cryo fluorescence data [5-7]. This amalgamation of techniques integrates 3D molecular localisation data with a high-resolution, $3 \mathrm{D}$ reconstruction of the cell.

At SiriusXT, in parallel with X-ray microscope development, we are also developing a correlative workflow compatible with our instrument.

The cryo-SXT imaging workflow is typically conducted in several stages:

1) preparation of biological samples. 2) placement of biological specimen on standard EM grid and vitrification by plunge-freezing. 3) cryo-light microscopy to: (a) verify success of vitrification process, (b) identify cells suitable for cryo-SXT and (c) to localise fluorescent molecules within the regions of interest for correlative microscopy. 4) cryo-SXT imaging 5) Overlay of cryo-fluorescence and cryo-SXT data.

For cryo-light microscopy, we have built our own cryo-correlative stage designed for an inverted $\mathrm{Ti}$ Eclipse microscope equipped with EMCCD (iXon 885 Andor), adopted with 100x, 0.9 N.A., 1mm long working distance air objective (Nikon Corporation).

We will present our cryo correlative workflow in detail, including results of cryo-light microscopy obtained with fluorescent and bright light transmitted modalities, as applied to a variety of biological specimens.
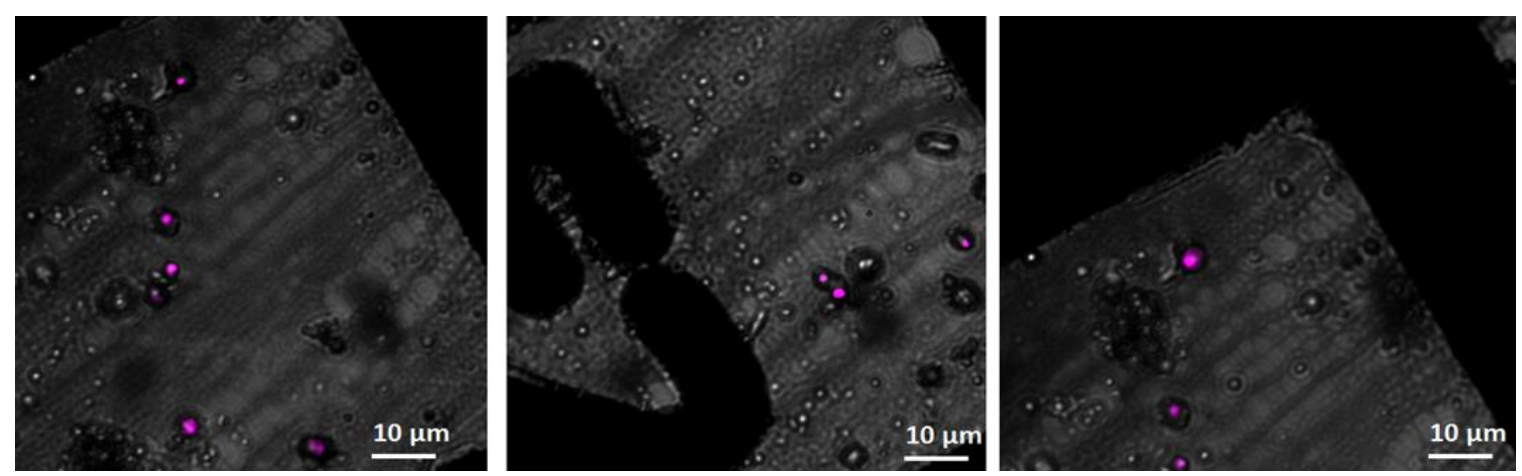

Figure 1. Cryo-fluorescent microscopy of Hoechst labelled Saccharomyces Cerevisiae 


\section{References}

[1] C.A. Larabell \& K.A. Nugent, Current Opinion in Structural Biology 20 (2010), p623.

[2] G. Schneider et al, Nature Methods 7 (2010), p985.

[3] R. Carzaniga et al, Protoplasma 251 (2014), p449.

[4] J.L. Carrascosa et al, Journal of Structural Biology 168 (2009), p234.

[5] B.P. Cinquin et al, Journal of Cellular Biochemistry 115 (2014), p209.

[6] R. Carzaniga et al, Methods in Cell Biology 124 (2014), p151.

[7] K.C. Dent et al, Methods in Cell Biology 124 (2014), p179. 